\title{
ABSORPTION CHARACTERISTICS OF DUST IN THE LYMAN CONTINUUM REGION
}

\author{
P. G. MEZGER, E. B. CHURCHWELL, and L. F. SMITH \\ Max-Planck-Institut für Radioastronomie, Bonn, F.R.G.
}

\begin{abstract}
The absorption cross-sections of dust grains in the Lyman continuum region are calculated based on integrated spectra of $\mathrm{OB}$ star clusters and observed values of the IR excess radiation from $\mathrm{H}$ II regions.

The IR excess radiation from $\mathrm{H}$ II regions, i.e., IR radiation in excess of that expected for heating by trapped Ly- $\alpha$ photons, is determined by both the absorption crosssections of dust grains and the radiation spectrum of the associated star cluster shortward and longward of the Lyman continuum limit. We compute integral spectral parameters of OB-star clusters and apply these to the problem of dust heating in $\mathrm{H}$ II regions. Comparison of our results with observations indicates that the effective absorption cross-section of dust in the Lyman-continuum region is about $50 \%$ of the extinction cross-section in the visual. Absorption of Lyman-continuum photons by dust grains appears to be selective, with an effective absorption cross-section shortward of $504 \AA$ about three times the effective absorption cross-section shortward of $912 \AA$.

Large values of IR excess radiation are to be expected for both dense and compact $\mathrm{H}$ II regions and for giant $\mathrm{H}$ II regions with large intrinsic stellar Lyman-continuum photon fluxes. At densities $n_{\mathrm{e}}>10^{5}$ to $10^{6} \mathrm{~cm}^{-3}, \mathrm{H}$ II regions will emit predominantly in the far IR as a result of opacity in the radio continuum and the fact that most of the Lyman-continuum photons are absorbed by dust. Values of (IR) $)_{\text {exc }} \sim 4$ appear to be typical for galactic H II regions; this (IR) exc corresponds to optical absorption depths of $<1$ shortward and longward of the Lyman-continuum limit.
\end{abstract}

We have given a fuller account in Astronomy and Astrophysics 32 (1974), p. 269.

\author{
P. G. Mezger \\ E. B. Churchwell \\ L. F. Smith \\ Max-Planck-Institut für Radioastronomie, \\ Auf dem Hügel 69, \\ 53 Bonn 1, F.R.G.
}

\section{DISCUSSION}

Wynn-Williams: I think there are dangers in using Hoffman, Frederick and Emery's data to derive far infrared luminosities, since they used a comparatively narrow bandwidth with the result that their fluxes depend strongly on the dust temperature in the source. If you use Harper and Low's or Emerson, Jennings and Moorwood's results there is a very low dispersion in the ratio of radio to infrared fluxes among the more luminous $\mathrm{H}_{\mathrm{II}}$ regions. 
Mezger: We multiplied the IR-flux densities given by Hoffman et al. by a factor of 4; this corresponds to a color temperature of the dust of about 70 to $80 \mathrm{~K}$. To my knowledge there are no indications that the color temperatures of dust vary strongly from $\mathrm{H}$ il region to Hil region. Most values of $(\text { IR })_{\text {exc }} \simeq$ $\simeq\left(4 \pi D^{2} F_{\mathrm{IR}} / N_{\mathrm{c}} h v_{\alpha}\right)-1$ fall within the range 1 to 4 ; however, some $\mathrm{H}$ II regions, notably those in the galactic center, have very high values of (IR) exc.

Zuckerman: I agree with Dr Mezger that when one looks carefully at Hil regions one finds that the apparently good correlation between far infrared luminosity and radio continuum flux breaks down. For example the Kleinman-Low nebula, NGC 2264 and DR21 (OH), all strong far infrared emitters, produce essentially no radio continuum flux.

Lequex: Could Dr Greenberg comment about the ratio of color excess in this region?

Greenberg: I chose not to discuss the problem of the very high albedo in the ultraviolet. I think this is a very confusing question. The albedo for these particles should be of the order of 0.5 , in the very, very far ultraviolet. 